\title{
Helical edge and surface states in HgTe quantum wells and bulk insulators
}

\author{
Xi Dai ${ }^{1,3}$, Taylor L. Hughes ${ }^{2}$, Xiao-Liang Qi $^{2}$, Zhong Fang ${ }^{1}$ and Shou-Cheng Zhang ${ }^{2}$ \\ 1 Beijing National Laboratory for Condensed Matter Physics, \\ Institute of Physics, Chinese Academy of Sciences, Beijing, 100080, China \\ 2 Department of Physics, McCullough Building, Stanford University, Stanford, CA 94305-4045 and \\ 3 Department of Physics, University of Hong Kong, Pokfulam Road, Hong Kong
}

\begin{abstract}
The quantum spin Hall (QSH) effect is the property of a new state of matter which preserves time-reversal, has an energy gap in the bulk, but has topologically robust gapless states at the edge. Recently, it has been shown that $\mathrm{HgTe}$ quantum wells realize this novel effect [1]. In this work, we start from realistic tight-binding models and demonstrate the existence of the helical edge states in HgTe quantum wells and calculate their physical properties. We also show that $3 \mathrm{~d} \mathrm{HgTe}$ is a topological insulator under uniaxial strain, and show that the surface states are described by single-component massless relativistic Dirac fermions in $2+1$ dimensions. Experimental predictions are made based on the quantitative results obtained from realistic calculations.
\end{abstract}

PACS numbers: 72.15Gd,73.63.Hs,75.47.-m,72.25.-b

Conventional insulators have a gap for all charge excitations and their physical properties are not sensitive to changes in the boundary conditions. Recently, a new class of quantum spin Hall(QSH) insulators has been proposed theoretically in both $2 \mathrm{~d}$ and $3 \mathrm{~d}[1,1,2,3,4,4,5]$. The QSH insulators are invariant under time reversal, have a charge excitation gap in the bulk, but have topologically protected gapless edge states that lie in the bulk insulating gap. This type of insulator is typically realized in spin-orbit coupled systems; and the corresponding edge states have a distinct helical property: states with one spin-polarization move around the sample edge in one direction, while states with the opposite spin-polarization move in the opposite direction [2, 6, 7]. The helical edge states are responsible for the intrinsic spin Hall effect in the insulating state $8,9,10,11]$. The edge states come in Kramers's doublets, and time reversal symmetry ensures the crossing of their energy levels at special points in the Brillouin zone. Because of this level crossing, the spectrum of a QSH insulator cannot be adiabatically deformed into that of a topologically trivial insulator without helical edge states; therefore, in this precise sense, the QSH insulators represent a topologically distinct new state of matter. In 3d the class of strong topological insulators has a similar distinction [4, 12] and is the natural generalization of the QSH insulator.

The study of the QSH effect in quasi- $2 \mathrm{~d} \mathrm{HgTe} / \mathrm{CdTe}$ quantum wells carried out in Ref. [1] is based on a simplified model obtained by $\mathbf{k} \cdot \mathbf{P}$ perturbation theory and the envelope function approximation. The conclusion of a topological quantum phase transition is reached based on a Dirac-type subband level crossing at the $\Gamma$ point. However, such a level crossing is not generic and could be avoided in the real system due to the bulk inversion asymmetry (BIA) of the zinc-blende lattice. Consequently, a more realistic study is necessary to obtain a better understanding of the QSH phase and the topological phase transition. In this paper, we study the subband structure and edge state properties of $\mathrm{HgTe} / \mathrm{CdTe}$ quantum wells using a realistic tight-binding(TB) model. The level crossing avoided at the $\Gamma$ point is recovered at several finite wavevectors. In other words, the phase transition between two different insulating regions remains robust despite inversion symmetry breaking. Furthermore, the topological nature of the QSH regime is demonstrated explicitly by studying the properties of the helical edge states in an open boundary system. We also apply the same realistic TB calculations to uniaxial strained $3 \mathrm{~d}$ $\mathrm{HgTe}$, and obtain the topologically non-trivial surface states. Thus, strained bulk HgTe is demonstrated to be a strong topological insulator. Ref. [12] investigates a class of models with bulk inversion symmetry which strictly speaking does not apply to $\mathrm{HgTe}$, however, adiabatic continuity is used to argue that strained bulk $\mathrm{HgTe}$ could be a strong topological insulator.

The CdTe and HgTe materials have the same zincblende lattice structure and are well-described by the same type of tight binding Hamiltonian, albeit with different parameters. This Hamiltonian includes two s-type orbitals and three p-type orbitals on each atom and reads

$$
\begin{aligned}
H= & \sum_{i \sigma \vec{R}} E_{i, a} a_{i \sigma \vec{R}}^{\dagger} a_{i \sigma \vec{R}}+\sum_{i \sigma \vec{R}} E_{i, c} c_{i \sigma, \vec{R}+\vec{d}}^{\dagger} c_{i \sigma, \vec{R}+\vec{d}}+\sum_{\vec{R}, \overrightarrow{R^{\prime}, \sigma i j}} V_{i, j} a_{i \sigma \vec{R}}^{\dagger} c_{i \sigma, \vec{R}+\vec{d}}+\text { H.C. } \\
& +\sum_{i j \sigma \sigma^{\prime} \vec{R}} \frac{4 \lambda_{a}}{\hbar} \vec{L}_{a, i j} \cdot \vec{S}_{a, \sigma \sigma^{\prime}} a_{i \sigma \vec{R}}^{\dagger} a_{j \sigma^{\prime} \vec{R}}+\sum_{i j \sigma \sigma^{\prime} \vec{R}} \frac{4 \lambda_{c}}{\hbar} \vec{L}_{c, i j} \cdot \vec{S}_{c, \sigma \sigma^{\prime}} c_{i \sigma \vec{R}+\vec{d}}^{\dagger} c_{j \sigma^{\prime} \vec{R}+\vec{d}}
\end{aligned}
$$


where $a_{i \sigma \vec{R}}^{\dagger}$ and $c_{i \sigma, \vec{R}+\vec{d}}^{\dagger}$ are the creation operators for electrons on the anion and cation sites respectively, $i=$ $\left(s, s^{*}, p_{x}, p_{y}, p_{z}\right)$ is the orbital index, and $\sigma$ is the spin index. $E_{i, a}, E_{i, b}$ and $V_{i, j}$ are the tight binding parameters defined by Slater and Koster [13]. Spin-orbit coupling is contained in the last two terms, and is represented by two coupling constants $\lambda_{a}$ and $\lambda_{b}$. The tight binding parameters are taken from Ref. [14] where they were determined by fitting to a first-principles calculation.

To obtain the boundary (edge or surface) states for quasi-2d $\mathrm{HgTe} / \mathrm{CdTe}$ quantum well structures and for the bulk 3d materials, we apply the Green's function method 15, 16] based on the TB model described above. For the quantum well system, we consider a symmetric $\mathrm{HgTe} / \mathrm{CdTe}$ hetero-structure with a fixed $N_{c}=8$ layers of CdTe surrounding a variable $N_{h}$ layers of $\mathrm{HgTe}$ on each side. In order to calculate the boundary states we choose open boundary conditions along the $x$-direction, periodic boundary conditions along the $y$-direction, and the open boundary condition along the $z$-direction which is the growth direction of the quantum well. For the bulk materials, surface states can be calculated for a semi-infinite system with open boundary conditions in one direction, such as [001], and periodic boundary conditions in the other two directions. The inverse Green's functions of both the quantum well and bulk materials can be written in a block tri-diagonal form as

$$
G^{-1}(z)=z-H=\left(\begin{array}{cccc}
z-H_{0} & C & 0 & 0 \\
C^{\dagger} & z-H_{0} & C & 0 \\
0 & C^{\dagger} & z-H_{0} & C \\
0 & 0 & C^{\dagger} & \cdots
\end{array}\right)
$$

where the diagonal block $H_{0}$ describes the Hamiltonian within the same "principal layer",15 that is, the layer along the x-direction with open boundary conditions for the quantum well case, or that along the only direction with open boundary condition for the bulk case. The off-diagonal block $C$ describes the coupling between two nearest-neighbor principal layers. To study the boundary states we only need $g_{i j}$, the Green's function on the boundary, where $i, j$ are the indices of the local basis on the boundary. This function is contained in the first diagonal block of the matrix $G(z)=(z-H)^{-1}$ and can be expressed in a recursive way as $g_{i j}^{(N)}=$ $\left(z-H_{0}-C g^{(N-1)} C^{\dagger}\right)_{i j}^{-1}$ with $g_{i j}^{(N)}$ denoting the boundary Green's function for a system with $N$ principal layers. The above recursive equations can be closed by the initial condition $g_{i j}^{(1)}=\left(z-H_{0}\right)^{-1}$, and we obtain $g_{i j}^{(N)}$ iteratively. Any physical observables projected onto the boundary are easily expressed using these Green's functions as $-\frac{1}{\pi} \int d \omega \sum_{i j} \operatorname{Img}_{i j}^{(N)}\left(\omega+i 0^{+}\right) O_{j i}=$ $-\frac{1}{\pi} \int d \omega \rho_{o}(\omega)$ where $\rho_{o}(\omega)$ is a type density of states and $i, j$ run over all the local basis states on the boundary. For example, $O^{c}=\sum_{i}|i><i|$, and $O^{s}=\sum_{i j} L_{i j}^{z}+S_{i j}^{z}$ generate the density of states for charge and spin on the boundary respectively. Notice here we generalize the def- inition of "spin" to include all the local angular momentum with real spin and angular momentum of the local basis. This method is easily generalized to study the interface states between two semi-infinite crystals, e.g., $\mathrm{HgTe}$ and $\mathrm{CdTe}$, which is essential in the present work as will be explained below.

As is well-known for HgTe quantum wells, the confinement effect along the $z$-direction opens a small gap around the Fermi level which makes the quantum well an insulator. By the analysis in Ref. [1] based on the $\mathbf{k} \cdot \mathbf{P}$ approximation, a quantum phase transition from a topologically trivial phase to a non-trivial phase (QSH phase) occurs for $\mathrm{CdTe} / \mathrm{HgTe}$ quantum-wells at some critical thickness of HgTe layer, which is signaled by a level crossing between the E1 and HH1 subbands. We study this transition using the more realistic full TB Hamiltonian given above. First we choose periodic boundary conditions in $x$ and $y$ to obtain the $2 \mathrm{~d}$ subband spectrum for different thicknesses $N_{h}$ of HgTe layers. In Fig 1 (b), we plot the subband spectrum at the $\Gamma$-point as a function of $\mathrm{HgTe}$ "half"-layers. Whereas Ref. [1] predicts that the subbands will cross, we find an anti-crossing at the $\Gamma$-point at a critical layer thickness $d_{c}=9 a$. This difference between the work of Ref.[1] and these TB results can be explained by the presence of BIA in the zinc-blende lattice which is ignored in the previous paper. From the $\mathbf{k} \cdot \mathbf{P}$ perspective, an additional term $H^{\prime}=C_{k} k_{z}\left\{J_{z},\left(J_{x}^{2}-J_{y}^{2}\right)\right\}$ is allowed in the bulk Hamiltonian once the point group symmetry is reduced by BIA to $D_{2 d}[18]$, with $J_{x}, J_{y}, J_{z}$ the spin- $3 / 2$ matrices. Despite being a $k_{z}$-dependent term in the bulk system, in the quantum well $H^{\prime}$ generates a constant term with $f_{i-}$ nite matrix elements connecting the $\mid E 1 \pm>$ subbands with the $\mid H H 1 \mp>$ subbands near the $\Gamma$-point, which can be derived following the effective $2 \mathrm{~d} \mathbf{k} \cdot \mathbf{P}$ approach of Ref. 1]. This term qualitatively affects the physics in exactly the manner predicted by the TB model.

Upon further investigation, we find that the inversion asymmetry shifts the crossing point from the $\Gamma$-point to eight non-zero $k$-points around it. In Fig:1 (a), we plot the subband spectrum at one of these crossing points $\mathbf{k}=(0.017,0.008) \frac{\pi}{a}$ where a clear level crossing between $E 1$ and $H H 1$ is observed. (Notice that away from $\Gamma$ point, the inversion asymmetry removes the double degeneracy as expected.) The other seven crossing points are determined by the point-group star of this one. We find that even though the level crossing at $\Gamma$ point is avoided, the gap-closing quantum phase transition between two insulating regimes predicted previously in the simplified model[1] still exists in the more realistic case.

After identifying the presence of the quantum phase transition, the next natural question is whether the topologically non-trivial phase on the thicker side of the transition predicted by the $\mathbf{k} \cdot \mathbf{P}$ calculation will survive the inversion symmetry breaking. The most convincing way to answer this question is to calculate the edge states directly. By the recursion method we obtain the charge and spin density of states defined above. The charge den- 
sity of states for a quantum well structure with 20 and 10 "half"-layers are plotted in Figs 2 (a) and (b) respectively. Sharp peaks appear in the gap for the quantum well with 20 half-layers and are absent in the 10 half-layer system. As required by time reversal symmetry (TRS), the energy levels at the $\Gamma$ point must be doubly degenerate, but can be split at finite $k_{y}$. The energy level splitting near $k_{y}=0$ as a function of $k_{y}$ is plotted in the inset of Fig. 2(a), which shows a perfect linear dispersion indicating a level crossing of the edge states at $k_{y}=0$. The spin densities of states for two $k_{y}$ 's with opposite signs are plotted in Fig. 2(c). If the chemical potential lies between the two peaks, only the lower branch of the edge states is occupied and a spin current will be carried by the edge states. With the recursion method we can also obtain the charge density of states on the inner layers away from the edge. In the inset of Fig. 2(c), we plot the height of the in-gap peak for $k_{y}=0$ on the different layers, which decays very fast from the edge and thus demonstrates that the in-gap peak is produced by the edge states. Finally, we plot the edge state dispersion in Fig. 2(d) with a color intensity plot generated from the density of states. We find that the edge states merge with the bulk states very quickly as $k_{y}$ moves away from the $\Gamma$-point. Consequently, whenever the chemical potential lies in the bulk gap, the only low-energy states crossing the Fermi level are one Kramers's pair of edge states. In other words, the low energy behavior of the quantum well in the bulk insulating region is described by an odd number of pairs of $1 \mathrm{~d}$ channels propagating on each edge. According to Ref $\underline{6}$, such a $1 d$ liquid is a "helical liquid," and cannot be realized in any pure $1 \mathrm{~d}$ system that preserves TRS. It can only exist as the edge theory of a $2 \mathrm{~d}$ QSH insulator. In this way, the results of our calculations provide convincing evidence that the $\mathrm{HgTe} / \mathrm{CdTe}$ quantum well is a QSH insulator characterized by a non-trivial $Z_{2}$ topological invariant [1, 2].

The same recursion method can also be applied for the $2 \mathrm{~d}$ surface states of strained $3 \mathrm{~d} \mathrm{HgTe}$, which was recently suggested to be topologically nontrivial 12]. However, the TB model (11) applied to a semi-infinite system with a $2 \mathrm{~d}$ surface always generates some surface states, even for a trivial band insulator, e.g., CdTe. 16] Physically, these surface states correspond to a dangling $s p^{3}$-hybridized bond at each surface atom, and are thus strongly dependent on the details of the surface physics, such as surface reconstruction and disorder. Since in the present paper we are only concerned about the topological properties, which are insensitive to the details of surface physics, we can choose a surface regularization that removes the trivial surface states and leaves only the topological ones. Therefore, we will focus on the interface between bulk $\mathrm{HgTe}$ and CdTe, where the dangling bonds of HgTe are coupled to CdTe so the trivial surface states vanish. Since CdTe can be adiabatically connected with vacuum by taking its band gap to infinity, the topological properties of $\mathrm{HgTe} / \mathrm{CdTe}$ interface are determined by $\mathrm{HgTe}$.

Since bulk HgTe is a semi-metal, we need to apply a

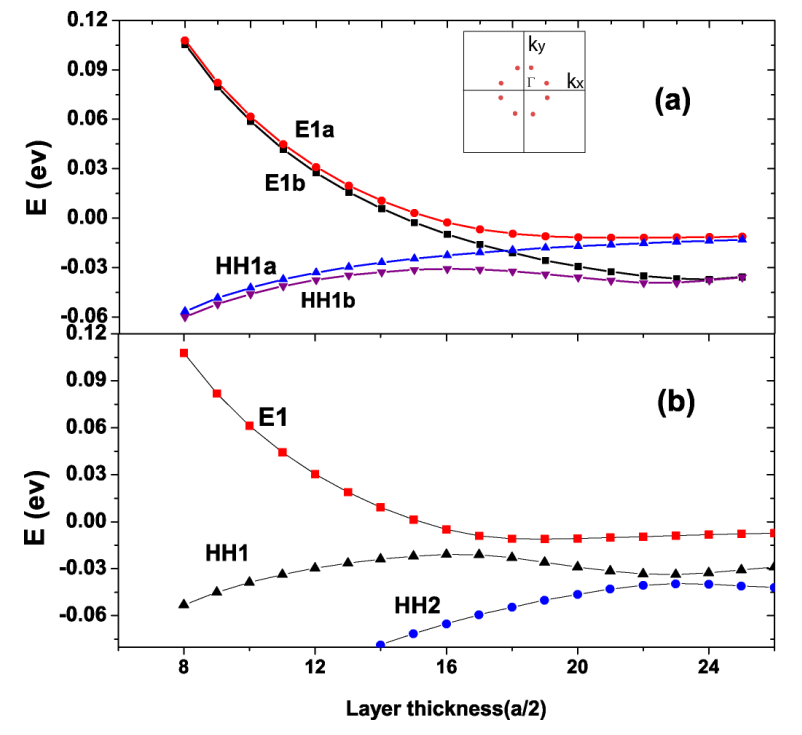

FIG. 1: (a) The subband splitting as the function of layer thickness at one of the crossing points $\mathbf{k}=$ $(0.017,0.008) \frac{\pi}{a}$. (Inset) Schematic diagram of all 8 crossing points in the $2 \mathrm{~d} \mathrm{BZ} \mathrm{(b)The} \mathrm{subband} \mathrm{splitting} \mathrm{as} \mathrm{the} \mathrm{func-}$ tion of layer thickness at the $\Gamma$ point.
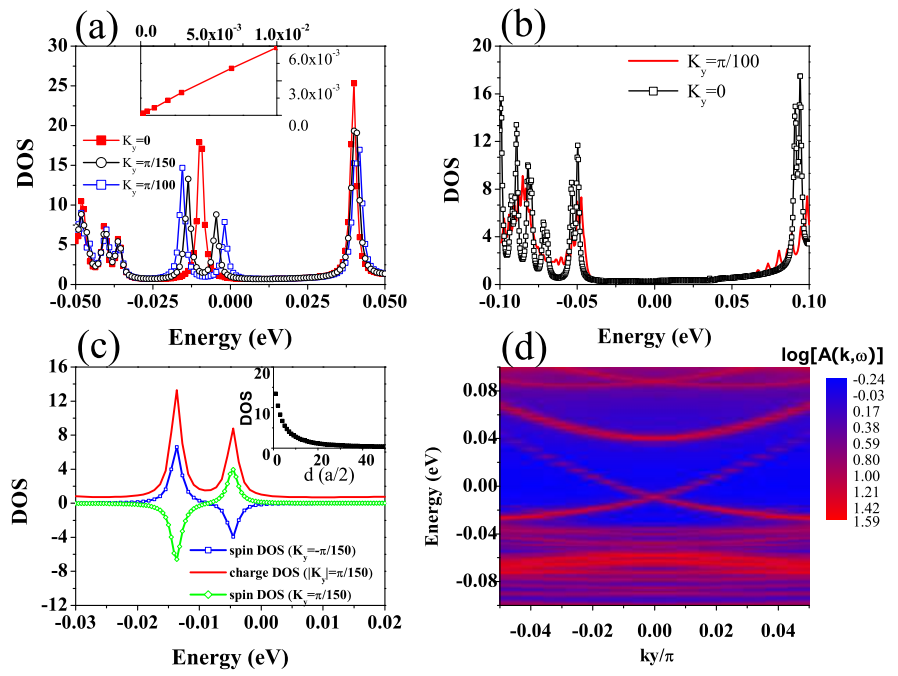

FIG. 2: (a)The density of states at the edge of the quantum well with layer thickness $d=20(a / 2)$. (The inset plot shows the linear energy splitting of the edge states in the very small region near $k_{y}=0$.)(b)The density of states at the edge of the quantum well with layer thickness $d=10(a / 2)$. (c) The spin density of states at the edge of the quantum well with thickness $d=20(a / 2)$. (The inset plot shows the decay of density of states at the peak energy of the spectra with $k_{y}=$ 0 .)(d)The intensity color plot on the energy-momentum plane for the density of states at the edge of the quantum well with thickness $d=20(a / 2)$. 


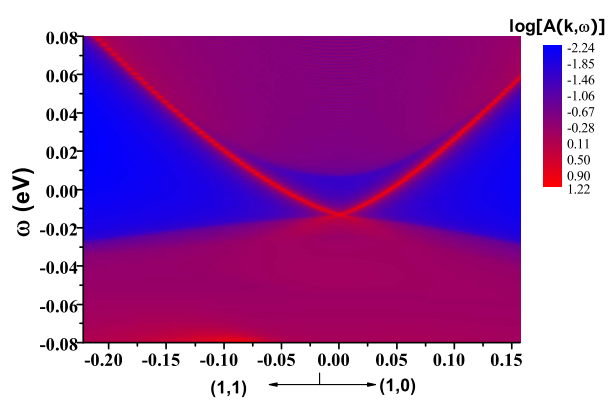

FIG. 3: Intensity color plot in the energy-momentum plane for the density of states at the $\mathrm{HgTe}-\mathrm{CdTe}$ interface. The uniaxial strain is applied along the [001] direction by choosing the $c / a$ ratio to be 0.98 and the $H g T e / C d T e$ interface is chosen along [100] direction.

small compressive strain, along say the [001] direction, to make it an insulator [11]. The tight binding parameters for the strained HgTe are obtained by fitting the LDA results from the plane wave pseudo potential method [17]. In the present paper, we apply the constrain along the [001] direction (z-direction) and locate the $\mathrm{HgTe} / \mathrm{CdTe}$ interface along both the [100] (x-direction) and [001] directions. And for both cases, we find non-trivial surface states. In Fig. 3 we plot the charge density of states in a color intensity plot. The single pair of surface states is clearly seen in the bulk insulating gap and they cross at the $\Gamma$-point. There are no other surface states in the entire zone. Any pure $2 \mathrm{~d}$ band theory that respects TRS must have the same number of Kramers's pairs on each of the time-reversal symmetric wave vectors because all of the $2 N$ energy bands must be paired on these wavevectors. As a result, the $2 \mathrm{~d}$ surface states of HgTe cannot emerge from any pure $2 \mathrm{~d}$ surface effect, and only as a consequence of bulk topology.

It is useful to try to understand this result from a continuum $\mathbf{k} \cdot \mathbf{P}$ perspective. HgTe has a non-trivial topological structure because the band-structure is only "inverted" near the $\Gamma$-point. The fact that an occupied band at this point has $\Gamma^{6}$ character means that the $Z_{2}$ invariant picks up an extra factor of $(-1)$ (if we ignore the small BIA) making it non-trivial. Due to strong orbital mixing the $\Gamma^{6}$ character is washed out as one moves away from the $\Gamma$-point and at the other special TR invariant points the "inverted" structure is absent. Therefore, we should be able to understand the topological properties from the band structure only near the $\Gamma$-point. The key point is to consider the full 6-band Kane model 19] instead of just the reduced 4-band Luttinger model [20]. If we only keep the bands in the Luttinger model the topological structure is absent. In the presence of uniaxial compressive strain along the (001) direction an insulating gap opens between the heavy-hole(HH) and light-hole( $\mathrm{LH})$ bands by pushing the $\mathrm{HH}$ band downward in energy. For a mo- ment we will ignore the $\mathrm{HH}$ band and focus only on the $\mathrm{LH}$ and $\Gamma^{6}(\mathrm{E})$ band. From the form of the Kane model, the coupling of the $\mathrm{LH}$ and $\mathrm{E}$ bands near the $\Gamma$-point is exactly a 3d anisotropic massive Dirac Hamiltonian if we ignore BIA and keep the leading order in $k$. The Dirac Hamiltonian preserves parity symmetry and we can label the bands by parity eigenvalues. Since the coupling is linear there must be one even (doubly degenerate) and one odd (doubly-degenerate) band. We expect that when the odd parity band lies below the even band then there will be a non-trivial $Z_{2}$ invariant which indicates an odd number of pairs of surface states that cross at TR invariant points [12]. The presence of the $\mathrm{HH}$ band will change the features of the spectrum but it does not change the presence of the surface states, or their protected crossing, as long as the strain induced gap is open. The system will remain a $3 \mathrm{~d}$ topological insulator when the $\mathrm{HH}$ band is coupled, and when BIA terms are added, as long as the bulk gap does not close. To show evidence of our statements we solve the 6-band Kane model on a cylinder. First we solve the model with the $\mathrm{HH}$ band completely decoupled from the LH and E bands (Fig. 4a). Here the $\mathrm{HH}$ band remains flat and is split from the $\mathrm{LH}$ band by the strain induced gap. In the gap there are clear, linearly dispersing surface states which traverse the gap between the LH and E bands. Nothing occurs at the other special points in the BZ, therefore this is a strong topological insulator. Turning on the coupling to the $\mathrm{HH}$ band changes features of the band structure but does not change the topology of the state since the gap between the LH and $\mathrm{HH}$ bands never closes. It is clear from Fig. 4b that even when the $\mathrm{HH}$ band is fully coupled the system is still a strong topological insulator with surface states crossing at $\Gamma$.

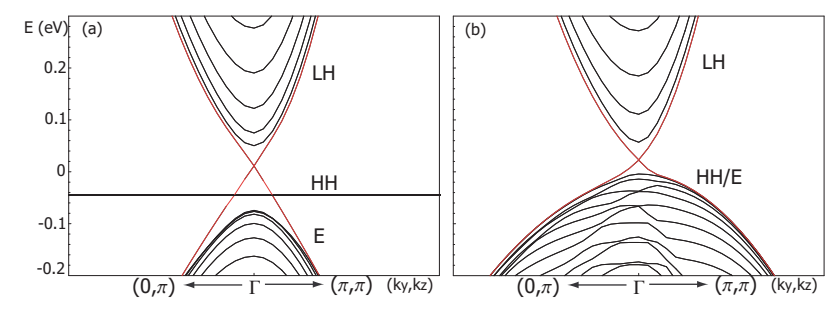

FIG. 4: Band structure near the $\Gamma$-point for (a) Decoupled $\mathrm{HH}$ band (b)Full HH band coupling. Surface sates are shown in red. Strain induced gap is artificially large so that surface states are clearly visible. Generally, topologically non-trivial surface states exist for any finite compressive strain.

This work is supported by the NSF under grant numbers DMR-0342832 and the US Department of Energy, Office of Basic Energy Sciences under contract DE-AC0376SF00515, the MARCO Center on Functional Engineered Nano Architectonics (FENA), and the Knowledge Innovation Project of the Chinese Academy of Sciences. 
[1] B. A. Bernevig, T. L. Hughes, and S.C. Zhang, Science 314, 1757 (2006).

[2] C. L. Kane and E. J. Mele, Phys. Rev. Lett. 95, 146802 (2005).

[3] B.A. Bernevig and S.C. Zhang, Phys. Rev. Lett. 96, 106802 (2006).

[4] L. Fu, C. Kane, and E. Mele, arxiv: cond-mat/0607699.

[5] J. E. Moore and L. Balents, arxiv: cond-mat/0607314.

[6] C. Wu, B.A. Bernevig, and S.C. Zhang, Phys. Rev. Lett. 96, 106401 (2006).

[7] C. Xu and J. Moore, Phys. Rev. B 73, 045322 (2006).

[8] S. Murakami, N. Nagaosa, and S.C. Zhang, Science 301, 1348 (2003).

[9] J. Sinova et. al., Phys. Rev. Lett. 92, 126603 (2004).

[10] S. Murakami, N. Nagaosa, and S.C. Zhang, Phys. Rev. B 69, 235206 (2004).

[11] S. Murakami, N. Nagaosa, and S.C. Zhang, Phys. Rev. Lett. 93, 156804 (2004).
[12] L. Fu and C. Kane, arxiv: cond-mat/0611341.

[13] J. Slater and G. Koster, Phys. Rev. B 94, 1498 (1954).

[14] A. Kobayashi, O. Sankey and J. Dow, Phys. Rev. B 25, 6367 (1982).

[15] J. K. M. S. I. Turek, V. Drchal and P. Weinberger, Electronic structure of disordered alloys, surfaces and interfaces, Kluwer Academic publishers, 1997.

[16] Garnett W. Bryant, Phys. Rev. B 35, 5547 (1987).

[17] Z. Fang and K. Terakura, J. Phys.: Cond. Mat. 14, 3001 (2002).

[18] R. Winkler, Spin-orbit coupling effects in twodimensional electron and hole systems, Springer Tracts in Modern Physics, 2003.

[19] E.O. Kane, J. Phys. Chem. Solids 1, 249 (1957).

[20] Y.R. Lin-Liu and L.J. Sham, Phys. Rev. B 32, 5561 (1985). 